\title{
An introduction to the dark energy problem
}

\author{
Antonio Dobado • Antonio L. Maroto
}

Received: 30 October 2007 / Accepted: 7 February 2008 / Published online: 25 April 2008

(C) Springer Science+Business Media B.V. 2008

\begin{abstract}
In this work we review briefly the origin and history of the cosmological constant and its recent reincarnation in the form of the dark energy component of the universe. We also comment on the fundamental problems associated to its existence and magnitude which require an urgent solution for the sake of the internal consistency of theoretical physics.
\end{abstract}

Keywords Dark energy · Cosmological constant

\section{Standard cosmology and Einstein's cosmological constant}

In 1915 Einstein introduced his famous field equations for gravity in the context of General Relativity, namely:

$R_{\mu \nu}-\frac{1}{2} R g_{\mu \nu}=8 \pi G T_{\mu \nu}$,

where $R_{\mu \nu}$ is the Ricci tensor and $R$ is the scalar curvature corresponding to the space-time metric $g_{\mu \nu}$ of signature $(+,-,-,-), G$ is the Newton constant and $T_{\mu \nu}$ is the matter energy-momentum tensor which for a perfect fluid has the general form:

$T_{\mu \nu}=(\rho+p) u_{\mu} u_{\nu}-p g_{\mu \nu}$,

with $\rho$ and $p$ being the energy density and pressure respectively and $u_{\mu}$ is the four-velocity. From the fluid proper frame this velocity is by definition $(1,0,0,0)$ (we are using

A. Dobado $(\varangle) \cdot$ A.L. Maroto

Departamento de Física Teórica, Universidad Complutense

de Madrid, 28040 Madrid, Spain

e-mail: dobado@ fis.ucm.es natural units where $c=\hbar=1$ ) and the energy-momentum tensor reads $T_{\mu \nu}=\operatorname{diag}(\rho, p, p, p)$.

Einstein field equations relate the space-time geometry (lhs) with its energy content (rhs) and probably are one of the most important landmarks in the whole history of physics. The covariant derivative of the geometrical side identically vanishes thus implying $D^{\mu} T_{\mu \nu}=0$ which are the equations of motion which must be supplemented with the matter equation of state. The Einstein equations describe properly at the classical level any known gravitational phenomena. Moreover they can be used to study the evolution of the universe as a whole. This can be done by assuming the Cosmological Principle which establishes that, at the largest scales, the universe is homogeneous and isotropic. In this case we have the so called Friedmann-Robertson-Walker (FRW) metric which can be written as:

$d s^{2}=d t^{2}-a^{2}(t)\left(\frac{d r^{2}}{1-k r^{2}}+r^{2}\left(d \theta^{2}+\sin ^{2} \theta\right) d \phi^{2}\right)$,

where the $k$ parameter can take the values 1,0 or -1 . By inserting this metric in the Einstein equations one gets two independent equations for the cosmological scale function $a(t)$. The Friedmann equation:

$H^{2} \equiv \frac{\dot{a}^{2}}{a^{2}}=\frac{8 \pi G}{3} \rho-\frac{k}{a^{2}}$,

where $H(t)=\dot{a}(t) / a(t)$ is the Hubble parameter and the deceleration equation:

$\frac{\ddot{a}}{a}=-\frac{4 \pi G}{3}(\rho+3 p)$.

The equation of motion (energy conservation) is just $\dot{\rho}=$ $-3 H(p+\rho)$. The equation of state is typically assumed 
to have the general form $p=w \rho$. Thus we have for example $w=0$ for non relativistic matter (dust) or $w=1 / 3$ for ultra-relativistic matter or radiation. Then the equation of motion can be easily integrated to find $\rho \sim a^{-3(1+w)}$. From the acceleration equation above it is clear that the sign of $\rho_{e}=\rho+3 p$ determines the acceleration state of the expansion, i.e., for $\rho_{e}>0$ the expansion would be decelerated whereas in the opposite case, the expansion rate would increase in time.

Assuming an universe dominated by non relativistic matter $(p=0)$ we have three different possibilities for its evolution. Introducing the critical density $\rho_{c} \equiv 3 H_{0}^{2} /(8 \pi G)$ (from now on we use for any dynamical quantity $X(t)$ the notation $X_{0} \equiv X\left(t_{0}\right)$, being $t_{0}$ the present time), we find: a recollapsing closed universe with $k=1$ for $\rho_{c}<\rho$, an open expanding universe with $k=-1$ for $\rho_{c}>\rho$, and the limiting case which has $k=0$ and is also expanding, flat, and has $\rho_{e}=\rho$. Notice that the expansion phases in all three cases are decelerated.

Einstein did not like this result at all since it implies the existence of a dynamical universe making not possible to have a static universe as was his expectation and the accepted paradigm for most people in those days. In fact, even before the above results were found, Einstein decided in 1917 to modify his field equations by introducing the cosmological constant term, since from his original field equations it was clear that a closed and static universe required to have $\rho_{e}=0$. The modified equations read:

$R_{\mu \nu}-\frac{1}{2} R g_{\mu \nu}-\Lambda g_{\mu \nu}=8 \pi G T_{\mu \nu}$,

where $\Lambda$ is the famous cosmological constant. Once this new term is included in the Einstein equations many things change. First of all Minkowski space is not a vacuum solution any more. The Newtonian potential, valid for low energy fields and low velocities, becomes:

$V(r)=-\frac{G M}{r}-\frac{\Lambda}{6} r^{2}$,

i.e., for $\Lambda>0$ the cosmological term produce some kind of anti gravity or repulsive gravity. By using the modified equations Einstein was able to find a closed and static solution with:

$\Lambda=\frac{3}{a^{2}}-8 \pi G \rho$.

However this solution has two important drawbacks. First it requires a fine tuning between density, cosmological constant and the universe radius. Second, and probably more important, the solution is not stable under small changes of the radius.

One useful and suggestive way to deal with the cosmological constant term consists in moving it from the lhs to the rhs of the Einstein field equations. This amounts to a redefinition of the energy-momentum tensor:

$T_{\mu \nu} \rightarrow \tilde{T}_{\mu \nu}=T_{\mu \nu}+T_{\Lambda \mu \nu}$,

where $T_{\Lambda \mu \nu} \equiv \Lambda g_{\mu \nu} /(8 \pi G)$, i.e., the new density and pressure are $\tilde{\rho}=\rho+\rho_{\Lambda}$ and $\tilde{p}=p+p_{\Lambda}$ with $\rho_{\Lambda}=\Lambda /(8 \pi G)$ and $p_{\Lambda}=-\rho_{\Lambda}$. Therefore the introduction of the cosmological constant is formally equivalent to the assumption of the existence of some kind of vacuum energy present even when there is no matter at all. However this vacuum energy (dark energy as denominated by $\mathrm{M}$. Turner) has very strange properties since it has $w_{\Lambda}=-1$. This means that for positive $\Lambda$ it has negative pressure and its effective density is $\rho_{e}=-2 \rho_{\Lambda}$, i.e., it is also negative and therefore produces accelerated expansion. In addition the $\rho_{\Lambda}$ does not change during the evolution of the universe as can be trivially obtained from the solution of the equation of motion given above. It is possible to define the so called cosmological parameter (which together with the value of the Hubble parameter $H_{0}$ completely specify a cosmological model), just by dividing the energy density of each component by $\rho_{c}$ defined above, i.e., $\Omega_{M, R}=\rho_{M, R} / \rho_{c}$ for matter or radiation, $\Omega_{\Lambda}=\rho_{\Lambda} / \rho_{c}$ and so on. From the Friedmann equation above (4), it is possible to find that $\Omega_{M}+\Omega_{\Lambda}+\Omega_{R}+\Omega_{K}=1$, where $\Omega_{K}=-k /\left(a_{0}^{2} H_{0}^{2}\right)$. All those facts are quite important in order to understand the present state of the universe as we will see in detail later.

Given the FRW metric it is not difficult to compute the physical or proper distance. For rays coming from a distant object at constant $\theta$ and $\phi$ at time $t$ we have:

$d(t)=\int_{0}^{r} \sqrt{g_{00}} d r^{\prime}=a(t) \int_{0}^{r} \frac{d r^{\prime}}{\sqrt{1-k r^{\prime 2}}}$.

For a photon emitted from this object at time $t$ and arriving now, i.e., at $t_{0}$, we have that the null geodesic equation $d s^{2}=0$ implies that the wavelength at origin $\lambda$ and the received wavelength $\lambda_{0}$ are related by $\lambda_{0} / \lambda=a(t) / a\left(t_{0}\right)$. It is customary to define the redshift parameter $z$ as $z \equiv$ $\left(\lambda_{0}-\lambda\right) / \lambda$ or $z=a_{0} / a-1$, i.e., it represents the fractional increase of wavelength in such a way that for $z>0$ we have redshift and an expanding universe and for $z<0$ we have blueshift and a contracting universe. From the observational point of view it is also interesting to introduce the so called luminosity distance. If $L$ is the luminosity of a distant object (total energy emitted per unit of time), the flux $\Phi$ (energy received per unit of area and time) is given by:

$\Phi=\frac{L}{4 \pi a_{0}^{2} r^{2}(1+z)^{2}}$,

where the two $1+z$ factors come from the photon energy redshift and the time dilatation between the emission and observation time respectively. The luminosity distance 
$d_{L}$ is defined so that $\Phi \equiv L /\left(4 \pi d_{L}^{2}\right)$ or in other words $d_{L}=a_{0} r(1+z)$. Now it is possible to expand this definition around $z=0$ for $z \ll 1$ to find:

$d_{L}=H_{0}^{-1} z\left(1+\frac{1-q_{0}}{2} z+\cdots\right)$,

where the deceleration parameter $q$ is defined as $q=$ $-\ddot{a} a / \dot{a}^{2}$. As commented above, for a matter dominated universe we would have $q_{0}>0$. For small enough $z$ we have:

$z \simeq H_{0} d_{L}$.

This is the famous Hubble law which was first found experimentally in the twenties by Wirtz and Hubble by plotting the redshift versus the luminosity distance of many galaxies around ours. Hubble found a positive value for $H_{0}$ given by $H_{0}=100 h \mathrm{~km} \mathrm{~s}^{-1} \mathrm{Mpc}^{-1}$ where $h$ is some number of order one. As $H_{0}$ is positive this shows that the universe is expanding which is probably one of the biggest scientific discoveries of all times. One of the important theoretical consequences of this fact is that, at least in principle, there is no need for introducing the cosmological constant. It is sad to think that if were not for the Einstein's prejudice about the static universe, he could have predicted this expansion years before its discovery. It is usually said that some time later Einstein declared that the introduction of the cosmological constant was the biggest blunder he ever made in his life.

\section{The accelerating universe and dark energy}

In 1998 two independent teams, the Supernova Cosmology Project (Perlmutter et al. (1999)) and the High- $z$ Supernovae Search Team (Riess et al. (1998)), extended the luminosity distance vs. redshift relation to higher redshifts $(z \lesssim 0.83)$. For that purpose they identified a new type of standard candle which was sufficiently bright to be seen from very long distances, namely a particular type of supernovae explosion known as Type Ia. This type of supernovae are found in binary systems in which one of the white dwarf stars exceeds the Chandrasekhar limits due to a accretion from its companion. Although the absolute luminosity is not the same for all SNIa, it is found that the duration of the explosion is related to the intrinsic luminosity and after appropriate rescaling and corrections a common light curve can be found for all SNIa.

Extending the Hubble diagram to high redshift allowed both teams to measure the deceleration parameter and, unexpectedly, they found a negative value for $q_{0}$, i.e., the expansion of the universe would be accelerating today rather than decelerating as one would expect in a matter dominated universe. A few years later, the Hubble Space Telescope (Riess et al. (2004)), identified 16 new SNIa at $z>1.25$.

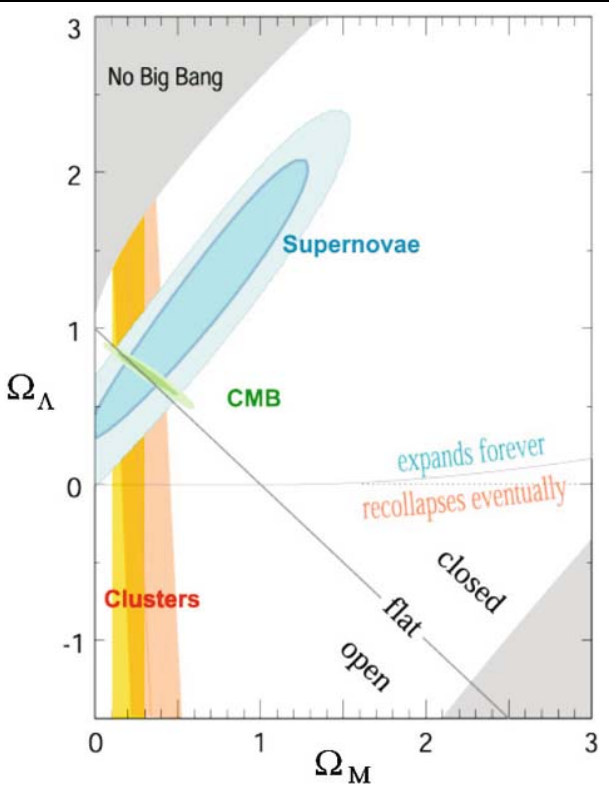

Fig. 1 Matter density $\Omega_{M}$ vs. vacuum energy density $\Omega_{\Lambda} 68 \%$ and 95\% C.L. contours for supernovae, cluster and CMB data (from Supernova/Acceleration Probe: SNAP collaboration (SNAP))

The new data allowed to confirm not only the present acceleration of the expansion rate, but also showed the existence of an deceleration-acceleration transition at redshift around $z_{c} \sim 0.5$. These results suggest a transition around $z_{c}$ from a standard matter dominated universe to a universe dominated by a new type of component with negative pressure (responsible for the acceleration) as required by (5).

The simplest explanation for that negative pressure fluid would be the introduction of a cosmological constant, with equation of state $w_{\Lambda}=-1$. Notice that in this case, the value of the cosmological constant is not tuned as in (8) in order to get an static Einstein universe, but instead its value would be responsible for an accelerated expansion.

In such a case, when combining the data from SNIa with Cosmic Microwave Background (CMB) anisotropies (Spergel et al. (2003)), it was possible to determine the cosmological abundances of the different components (see Fig. 1). Thus a good fit to the cosmological observations would correspond to the so called concordance model, i.e., a $\Lambda$ CDM model with $\Omega_{M}=0.27 \pm 0.04$ and $\Omega_{\Lambda}=0.73$ \pm 0.04 . In other words, the present universe would be mainly made of matter and this new form of energy with negative pressure.

In the last years further observational evidence, independent of SNIa observations have been obtained which supports the existence of dark energy. In general, dark energy is believed to be a weakly coupled component, its only effects being of gravitational nature which makes its direct detection extremely difficult. So far the observable effects of dark energy have been classified mainly in two classes: modifications of the redshift-distance relation which have 
Fig. 2 Evolution of the energy density of the different components vs. photon temperature for radiation, matter and cosmological constant. The circled area corresponds to the present time

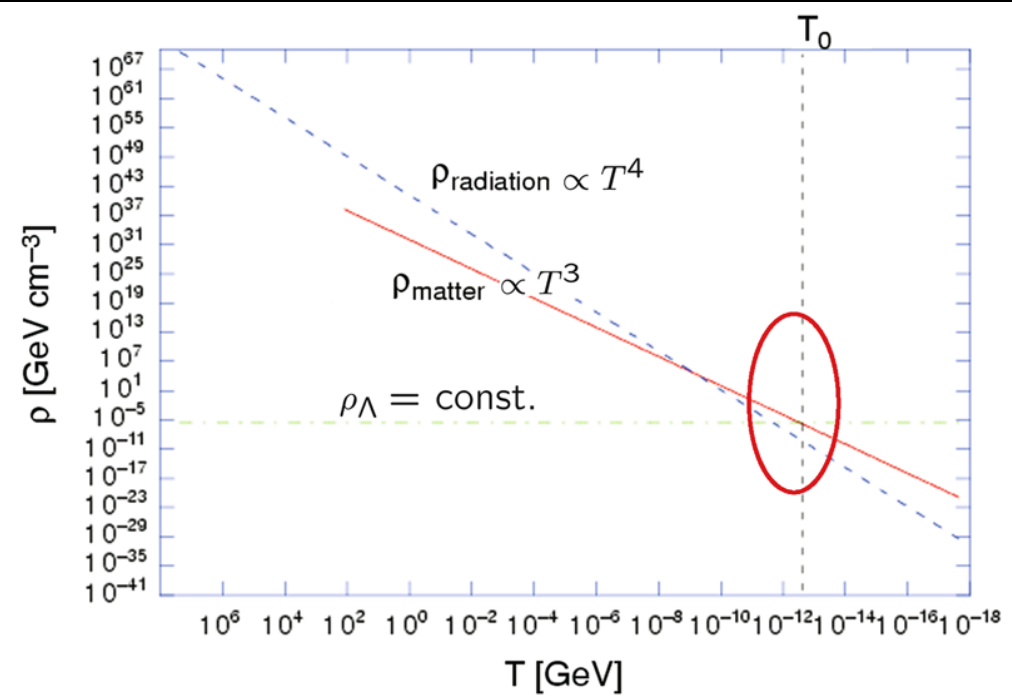

been observed not only through SNIa, but also by means of the so called baryon acoustic oscillations found in the largescale distribution of galaxies by the Sloan Digital Sky Survey and $2 \mathrm{dF}$ collaborations (Eisenstein et al. (2005); Cole et al. (2005)). On the other hand, we have the effects on the growth of structure, which could be measured through observations of galaxy clustering, weak lensing or the integrated Sachs-Wolfe effect on CMB anisotropies. These methods are currently in progress and new surveys are planned to develop in the next decade (Trotta and Bower (2006)).

\section{The cosmological constant problem and theoretical alternatives}

As we have seen, introducing a cosmological constant provides a simple explanation for the current state of accelerated expansion of the universe. However, a closer look at this solution reveals some unpleasant features which we will discuss in this section.

The observational value $\Omega_{\Lambda}=0.73$ means that the energy density of the cosmological constant is of the order of the critical density, i.e., $\rho_{\Lambda} \simeq\left(10^{-3} \mathrm{eV}\right)^{4}$. On the other hand the value of the Newton's constant is $G \simeq\left(10^{19} \mathrm{GeV}\right)^{-2}$ in natural units. In other words, if the cosmological constant is a true fundamental constant of nature, the gravitational interaction described by Einstein equations plus cosmological constant (6) would be controlled by two dimensional constants whose scales $\sim 10^{19} \mathrm{GeV}$ and $\sim 10^{-3} \mathrm{eV}$ are separated by more than 30 orders of magnitude. Explaining such an enormous difference is called the cosmological constant problem.

A possible solution is that $\Lambda$ is not a true constant of nature but some sort of effective term generated in the Einstein equations by other physics. Thus for example, the vacuum energy of all quantum fields present in the universe would contribute as $\rho_{\Lambda} \sim \int_{0}^{M_{*}} d^{3} k k$, with $M_{*}$ the ultraviolet cutoff of the theory. However the problem is not solved in this way since we typically have $M_{*} \gg 10^{-3} \mathrm{eV}$. There are other candidates to dark energy apart from the cosmological constant in the literature. Thus, it has been proposed that dark energy could be identified with the energy density of a dynamical scalar field (quintessence) (Copeland et al. (2006)). Such models could exhibit equations of state today which deviate from $w=-1$ and could be discriminated from a cosmological constant by future observations. However these models require appropriate potential terms whose scales have to be fine tuned, in a similar way to the value of the cosmological constant, in order for the accelerated period to start at the correct time.

The dark energy problem can also be seen from a different perspective. Since the time evolution of matter and cosmological constant are very different (see Fig. 2), the fact that today they have comparable values suggests that either we are living a sort of cosmic coincidence, without deeper explanation, or there is a strong relationship between the origin and evolution of the different components of the universe. In any case, these problems show why understanding the nature of dark energy has become one of the most important open questions in theoretical physics.

Acknowledgements This work has been partially supported by the DGICYT (Spain) under the project numbers FPA 2004-02602 and FPA 2005-02327 and by the Universidad Complutense/CAM: project number 910309. A.D. thanks Ana I. Gómez de Castro for her kind invitation to participate in the NUVA Conference.

\section{References}

Cole, S., et al.: Mon. Not. R. Astron. Soc. 362, 505 (2005) 
Copeland, E.J., Sami, M., Tsujikawa, S.: Int. J. Mod. Phys. D 15, 1753 (2006)

Eisenstein, D.J., et al.: Astrophys. J. 633, 560 (2005)

Perlmutter, S., et al.: Astrophys. J. 517, 565 (1999)

Riess, A.G., et al.: Astron. J. 116, 1009 (1998)
Riess, A.G., et al.: Astrophys. J. 607, 665 (2004)

Spergel, D.N., et al.: Astrophys. J. Suppl. Ser. 148, 175 (2003). astro$\mathrm{ph} / 0603449$

Trotta, R., Bower, R.: Astron. Geophys. 47, 4:20-4:27 (2006) 